\title{
SOERGEL CALCULUS AND SCHUBERT CALCULUS
}

\author{
XUHUA HE ${ }^{1, a}$ AND GEORDIE WILLIAMSON ${ }^{2, b}$
}

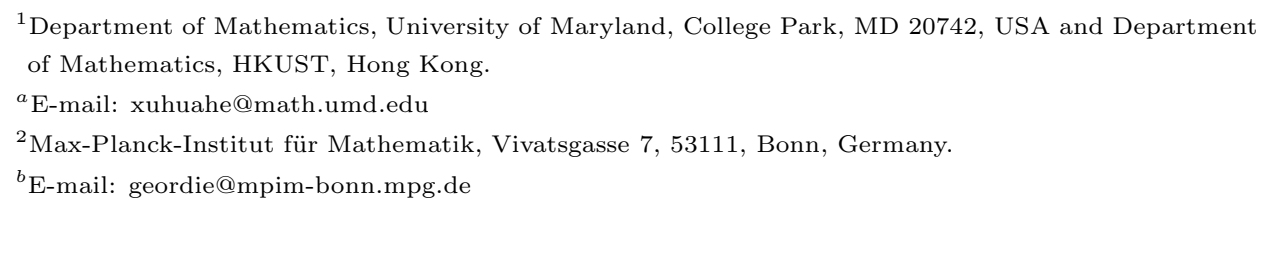

Abstract

We reduce some key calculations of compositions of morphisms between Soergel bimodules ("Soergel calculus") to calculations in the nil Hecke ring ("Schubert calculus"). This formula has several applications in modular representation theory.

\section{Introduction}

Determining the modular irreducible characters of the symmetric groups and of simple groups of Lie type is a major open problem in representation theory. Recently it has been discovered that there are certain hidden monoidal categories lurking "behind" or "inside" these categories of representations. The two most prominent examples of such categories are the Khovanov-Lauda-Rouquier categorifications of modules over Kac-Moody Lie algebras and module categories for Soergel bimodules (which categorify modules for Weyl groups or their associated Hecke algebras).

In this paper we concentrate on the monoidal category of Soergel bimodules. Although the definition of the category of Soergel bimodules is elementary (as a full subcategory of bimodules over a polynomial ring), calculations can be prohibitively difficult. Performing such calculations is important, as they often provide a means of calculating an irreducible character or a decomposition number. This is a theorem for rational representations

Received May 5, 2016 and in revised form May 11, 2016.

AMS Subject Classification: 20C20, 20C08, 20C33, 55N33, $14 \mathrm{~N} 15$.

Key words and phrases: Soergel bimodules, intersection forms, nil Hecke ring, Schubert calculus. 
of algebraic groups (if the characteristic is larger than the Coxeter number) via results of Soergel [18] and Fiebig [8]. A conjecture of Riche and the second author [17, Conjecture 1.6] would imply that these calculations also give all characters of tilting modules for algebraic groups, and hence all decomposition numbers for symmetric groups (this conjecture is a theorem for $G L_{n}$ in charateristic $p>n[17$, Theorem 1.8]). The reader is referred to the introductions of [11] and [17] for a more detailed survey of the connections between Soergel bimodules and modular representation theory.

Progress on the problem of performing calculations with Soergel bimodules has been made by Libedinsky [14], Elias-Khovanov [4], Elias [3] and Elias-Williamson [6] culminating in a presentation of the monoidal category of Soergel bimodules by generators and relations. The description is diagrammatic and has led to progress on understanding the category of Soergel bimodules and its module categories. Though much simpler than calculations in bimodules, these diagrammatic calculations can still be very difficult.

In particular, it is desirable to find additional simplifications that make calculations more feasible. In this paper we take the first step in this direction. The classes of indecomposable Soergel bimodules in the Grothendieck group are controlled by certain integral symmetric forms known as "intersection forms". For the above applications to modular representation theory it is important to calculate the ranks of these matrices modulo a prime number $p$. In this paper we explain that a subset of the entries of these matrices are canonical, and are given by a simple formula in the nil Hecke ring (see Theorem 5.1). Let us emphasise that our formula does in general determine the intersection form entirely. However we still feel our formula is a significant simplification, and we believe our formula will have other applications. In the final section of this paper we show that our formula can be used to easily rederive interesting examples discovered by Kashiwara-Saito and Braden. In [22] the second author uses this formula to construct many more such examples, and deduces that the exceptional characteristics occurring in Lusztig's conjectured character formula grow exponentially in the rank.

The authors have the optimistic hope that one should be able to reduce all essential calculations amongst Soergel bimodules to calculations in the nil Hecke ring. That this is in principle possible is evidenced by the work of Dyer [1]. Our goal is explicit formulas that allow us to calculate the 
characters of the indecomposable Soergel bimodules algorithmically. This paper can be seen as a first step in this direction.

\section{Background}

2.1. Let $S$ be a finite set and $\left(m_{s t}\right)_{s, t \in S}$ be a matrix with entries in $\mathbb{N} \cup\{\infty\}$ such that $m_{s s}=1$ and $m_{s t}=m_{t s} \geq 2$ for all $s \neq t$. Let $W$ be a group generated by $S$ with relations $(s t)^{m_{s t}}=1$ for $s, t \in S$ with $m_{s, t}<\infty$. We say that $(W, S)$ is a Coxeter system and $W$ a Coxeter group. The Coxeter group $W$ is equipped with the length function $\ell: W \rightarrow \mathbb{N}$ and the Bruhat order $\leq$.

An expression is a finite sequence of elements of $S$. We denote by

$$
\operatorname{Ex}(S)=\sqcup_{i \in \mathbb{N}} S^{i}
$$

the set of all expressions. Let $\underline{w}=\left(s_{1}, s_{2}, \ldots, s_{m}\right) \in \operatorname{Ex}(\mathrm{S})$. The length $\ell(\underline{w})$ of $\underline{w}$ is $m$. A subexpression of $\underline{w}$ is a sequence $\left(s_{1}^{e_{1}}, s_{2}^{e_{2}}, \ldots, s_{m}^{e_{m}}\right)$, where $e_{i} \in\{0,1\}$ for all $i$. We call $\underline{e}$ the associated 01 -sequence and simply write $\left(s_{1}^{e_{1}}, s_{2}^{e_{2}}, \ldots, s_{m}^{e_{m}}\right)$ as $\underline{w} \underline{e}$.

The group multiplication gives a natural map

$$
\operatorname{Ex}(\mathrm{S}) \rightarrow \mathrm{W}, \quad \underline{\mathrm{w}} \mapsto \underline{\mathrm{w}}_{\bullet},
$$

where $\underline{w}_{\bullet}=s_{1} s_{2} \cdots s_{m}$ for $\underline{w}=\left(s_{1}, s_{2}, \ldots, s_{m}\right)$. Notice that $\ell\left(\underline{w}_{\bullet}\right) \leq \ell(\underline{w})$. If equality holds, then we call $\underline{w}$ a reduced expression.

Given a subexpression $\underline{w}^{\underline{e}}$ of $\underline{w}$ we set $\left(\underline{w}^{\underline{e}}\right) \bullet=s_{1}^{e_{1}} s_{2}^{e_{2}} \ldots s_{m}^{e_{m}}$.

2.2. Now we recall the Demazure product.

Let $x, y \in W$. By [9, Lemma 1], the set $\{u v ; u \leq x, v \leq y\}$ contains a unique maximal element. We denote this element by $x * y$ and call it the Demazure product of $x$ and $y$. Then

$$
\{u v ; u \leq x, v \leq y\}=\{w \in W ; w \leq x * y\}
$$

In particular, if $x^{\prime} \leq x$ and $y^{\prime} \leq y$, then $\left(x^{\prime}\right) *\left(y^{\prime}\right) \leq x * y$. 
The operator $*$ gives a monoidal structure on $W$. This monoidal structure gives another natural map

$$
\operatorname{Ex}(\mathrm{S}) \rightarrow \mathrm{W}, \quad \underline{\mathrm{w}} \mapsto \underline{\mathrm{w}}_{*},
$$

where $\underline{w}_{*}=s_{1} * s_{2} * \cdots * s_{m}$ for $\underline{w}=\left(s_{1}, s_{2}, \ldots, s_{m}\right)$.

By definition, $\left(\underline{w}^{\underline{e}}\right) \bullet \leq \underline{w}_{*}$ for any 01-sequence $\underline{e}$. In particular $\underline{w}_{\bullet} \leq \underline{w}_{*}$.

2.3. Given an expression $\underline{w}=\left(s_{1}, s_{2}, \ldots, s_{m}\right)$, a 01 -sequence $\underline{e}=\left(e_{1}, e_{2}, \ldots\right.$, $\left.e_{m}\right)$ and $0 \leq k \leq m$, we set $\underline{w}_{\leq k}=\left(s_{1}, \ldots, s_{k}\right), \underline{e}_{\leq k}=\left(e_{1}, \ldots, e_{k}\right)$ and

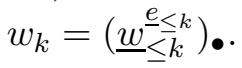

We define the decorated sequence $\left(d_{1} e_{1}, d_{2} e_{2}, \ldots, d_{m} e_{m}\right)$ associated to $(\underline{w}, \underline{e})$. Here $d_{i} \in\{U, D\}$ is the decoration to $e_{i}$. (Here $U$ stands for "Up" and $D$ stands for "Down".) The decoration is defined as follows. For any $i$,

$$
d_{i}= \begin{cases}U & \text { if } w_{i-1} s_{i}>w_{i-1} \\ D & \text { if } w_{i-1} s_{i}<w_{i-1}\end{cases}
$$

It will often be convenient to view $\underline{e}$ as the string

$$
\left(d_{1} e_{1}, d_{2} e_{2}, \ldots, d_{m} e_{m}\right)
$$

in the symbols $U 0, U 1, D 0, D 1$. As the decoration is determined by $\underline{e}$ and $\underline{w}$, this is no more information. The defect of $\underline{e}$ is defined to be

$$
\operatorname{df}(\underline{\mathrm{e}}):=\sharp\left\{\mathrm{i} ; \mathrm{d}_{\mathrm{i}} \mathrm{e}_{\mathrm{i}}=\mathrm{U} 0\right\}-\sharp\left\{\mathrm{i} ; \mathrm{d}_{\mathrm{i}} \mathrm{e}_{\mathrm{i}}=\mathrm{D} 0\right\} .
$$

2.4. The Hecke algebra $\mathcal{H}$ of $W$ is the free $\mathbb{Z}\left[v, v^{-1}\right]$-algebra with basis $H_{w}$ for $w \in W$ and multiplication given by

$$
\begin{gathered}
H_{x} H_{y}=H_{x y}, \quad \text { if } \ell(x y)=\ell(x)+\ell(y) ; \\
\left(H_{s}+v\right)\left(H_{s}-v^{-1}\right)=0, \quad \text { for } s \in S .
\end{gathered}
$$

For $\underline{w}=\left(s_{1}, \ldots, s_{m}\right) \in \operatorname{Ex}(\mathrm{S})$, we set $H_{\underline{w}}=H_{s_{1}} \cdots H_{s_{m}}$. Then $H_{\underline{w}}=$ $H_{\underline{w}}$. if $\underline{w}$ is a reduced expression.

The Hecke algebra $\mathcal{H}$ has a $\mathbb{Z}$-linear bar involution ${ }^{-}: \mathcal{H} \rightarrow \mathcal{H}$ sending $v$ to $v^{-1}$ and $H_{w}$ to $H_{w^{-1}}^{-1}$ for all $w \in W$. For any $w \in W$, the Kazhdan- 
Lusztig element $\underline{H}_{w}$ is the unique bar-invariant (i.e. $\bar{h}=h$ ) element in $H_{w}+\sum_{x<w} v \mathbb{Z}[v] H_{x}$. The elements $\left\{\underline{H}_{w} \mid w \in W\right\}$ form a basis of $\mathcal{H}$.

For $\underline{w}=\left(s_{1}, \ldots, s_{m}\right) \in \operatorname{Ex}(\mathrm{S})$, we set $\underline{H}_{w}=\underline{H}_{s_{1}} \cdots \underline{H}_{s_{m}}$.

2.5. We fix a realization (in the sense of $[6, \S 3.1]$ ) $\mathfrak{h}$ of $W$ over a commutative ring $\mathbb{k}$. Recall that this consists of a free and finitely generated $\mathbb{k}$-module $\mathfrak{h}$ together with subsets

$$
\left\{\alpha_{s}\right\}_{s \in S} \subset \mathfrak{h}^{*} \text { and }\left\{\alpha_{s}^{\vee}\right\}_{s \in S} \subset \mathfrak{h}
$$

of "roots" and "coroots" such that $\left\langle\alpha_{s}, \alpha_{s}^{\vee}\right\rangle=2$ for all $s \in S$ and the formulas (for $s \in S$ and $v \in \mathfrak{h}$ )

$$
s(v):=v-\left\langle\alpha_{s}, v\right\rangle \alpha_{s}^{\vee}
$$

define an action of $W$ on $\mathfrak{h}$.

For simplicity in this paper we will assume that one of the following two assumptions is satisfied:

(1) $\mathbb{k}=\mathbb{R}$ and $\mathfrak{h}$ is the geometric representation of $W$ (defined for example in [10, Section 5.3]);

$(2) \mathfrak{h}$ is obtained by extension of scalars from a realization defined over $\mathbb{Z}$ for which the matrix $\left(\left\langle\alpha_{s}^{\vee}, \alpha_{t}\right\rangle\right)_{s, t \in S}$ is a generalized Cartan matrix (in the sense of [12, Chapters 1 and 3]).

Additionally, we assume that the maps $\alpha_{s}: \mathfrak{h} \rightarrow \mathbb{k}$ and $\alpha_{s}^{\vee}: \mathfrak{h}^{*} \rightarrow \mathbb{k}$ are surjective. (This condition is called Demazure surjectivity in [6]. It is automatic if 2 is invertible in $\mathbb{k}$.)

Remark 2.1. It is possible (and can be interesting, see [2]) to consider more general realizations, however this can introduce extra subtleties. For example one may not have a good notion of positive roots and Demazure operators need not satisfy the braid relations. It is for this reason that we make the assumptions above.

We denote by $R=S\left(\mathfrak{h}^{*}\right)$ the symmetric algebra of $\mathfrak{h}^{*}$ over $\mathbb{k}$. We view $R$ as a graded $\mathbb{k}$-algebra with $\operatorname{deg} \mathfrak{h}^{*}=2$. Because $W$ acts on $\mathfrak{h}^{*}$, it also 
acts on $R$ by functoriality. For any $s \in S$ we define the Demazure operator $\partial_{s}: R \rightarrow R[-2]$ by

$$
\partial_{s}(f)=\frac{f-s f}{\alpha_{s}} .
$$

(This is well defined under our assumptions on $\mathfrak{h}$, see [6, §3.3].)

2.6. An $S$-graph is a finite, decorated, planar graph with boundary $\Gamma$ satisfying:

(1) The graph $\Gamma$ is properly embedded in the planar strip $\mathbb{R} \times[0,1]$.

(2) The edges of $\Gamma$ are colored by $S$ and all vertices are of the following types:

(i) univalent vertices ("dots"):

(ii) trivalent vertices:

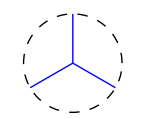

(iii) $2 m_{s t}$-valent vertices:

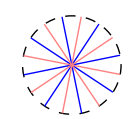

(We require that there are exactly $2 m_{s t}$ edges originating from the vertex, and that they are alternately colored $s$ and $t$ around the vertex. For example, the pictured example has $m_{s t}=8$.)

(3) The regions of $\Gamma$ may be decorated by boxes containing homogenous elements of $R$. (The regions of an $S$-graph are by definition the connected components of the complement of $\Gamma$ in $\mathbb{R} \times[0,1]$.)

Here is an example of an $S$-graph (with $m_{s, t}=5, m_{s, u}=2, m_{u, t}=3$ ):

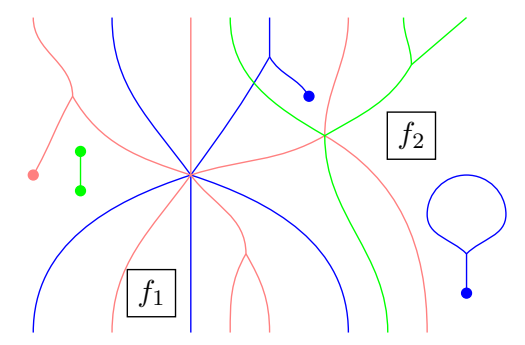

where $f_{i} \in R$ are homogenous polynomials. We define the degree of an $S$-graph as the sum over the degrees of its vertices and boxes, where each box has degree equal to the degree of the corresponding element of $R$, and 
the vertices have degrees given by the following rule: dots have degree 1 , trivalent vertices have degree -1 and $2 m_{s t}$-valent vertices have degree 0 . For example, the degree of the $S$-graph above is $-1+1-1+1-1-1+1+1+$ $1-1+\operatorname{deg} f_{2}+\operatorname{deg} f_{2}=\operatorname{deg} f_{1}+\operatorname{deg} f_{2}$.

The boundary points of any $S$-graph on $\mathbb{R} \times\{0\}$ and on $\mathbb{R} \times\{1\}$ gives two sequences of colored points, and hence two elements in $\operatorname{Ex}(\mathrm{S})$. We call these two sequences the bottom boundary and top boundary. For example, the bottom (resp. top) boundary of the $S$-graph above is $(s, t, s, t, t, s, u, t)$ (resp. $(t, s, t, u, s, t, u, u)$ ).

2.7. We now define the diagrammatic category of Soergel bimodules. Much greater detail and generality can be found in [3, 6]. The important case of $W$ of type $A$ is discussed in detail in [4]. Our intention is to give the reader a summary.

Let $\mathbb{S} \mathcal{D}$ be the monoidal category whose objects are $\underline{w} \in \operatorname{Ex}(\mathrm{S})$. For any $\underline{x}, \underline{y} \in \operatorname{Ex}(\mathrm{S}), \operatorname{Hom}_{\mathbb{S} \mathcal{D}}(\underline{\mathrm{x}}, \underline{\mathrm{y}})$ is defined to be the free $R$-module generated by isotopy classes of $S$-graphs with bottom boundary $\underline{x}$ and top boundary $\underline{y}$, modulo the local relations below. The structure of a monoidal category is induced by horizontal and vertical concatenation of diagrams.

Here are the relations. We use the coloring $s$ and $t$.

\subsubsection{Frobenius unit.}

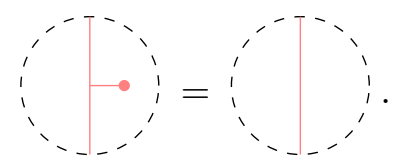

\subsubsection{Frobenius associativity.}

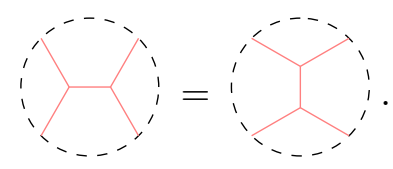

\subsubsection{Needle relation.}

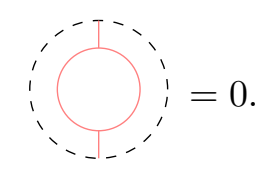




\subsubsection{Barbell relation.}

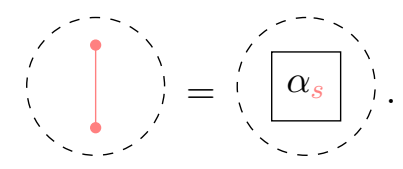

2.7.5. Nil Hecke relation.

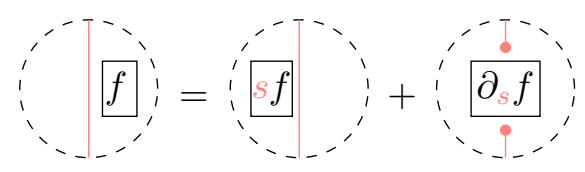

(See $\S 2.5$ for the definition of $\partial_{s}$.)

\subsubsection{Two-color associativity.}

We give the first three cases, i.e. $m_{s t}=2,3,4$. The reader can probably guess the general form (see [3] (6.12) for all the details).

$$
m_{s t}=2\left(\text { type } A_{1} \times A_{1}\right) \text { : }
$$

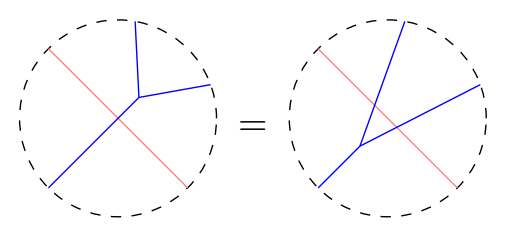

$$
m_{s t}=3\left(\text { type } A_{2}\right) \text { : }
$$

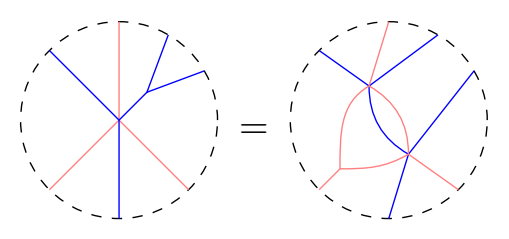

$$
m_{s t}=3\left(\text { type } B_{2}\right) \text { : }
$$

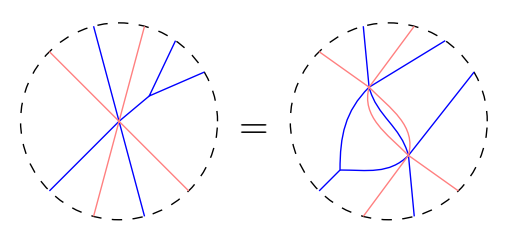




\subsubsection{The Jones-Wenzl relation.}

Elias' Jones-Wenzl relation expresses a dotted $2 m_{s t}$-vertex

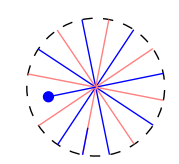

as a linear combination of diagrams consisting only of dots and trivalent vertices. We will not give the general form of the relation here, as the determination of the coefficients is complicated. (To understand the results of this paper, explicit knowledge of only the simplest coefficients is necessary.) We give the details when $m_{s t}=2,3,4$ and refer the reader to [3] for more information.

$$
m_{s t}=2\left(\text { type } A_{1} \times A_{1}\right) \text { : }
$$

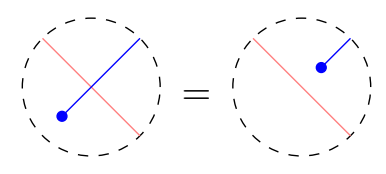

$$
m_{s t}=3\left(\text { type } A_{2}\right) \text { : }
$$

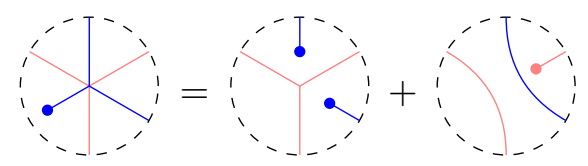

$m_{s t}=4$ (type $B_{2}$ ). Here there are two natural choices of realization, and this affects the coefficients in the Jones-Wenzl relation. Assume first that we are using the symmetric "geometric" realization, so that

$$
\left\langle\alpha_{s}^{\vee}, \alpha_{t}\right\rangle=\left\langle\alpha_{t}^{\vee}, \alpha_{s}\right\rangle=-\sqrt{2}
$$

Here a Jones-Wenzl relation takes the form

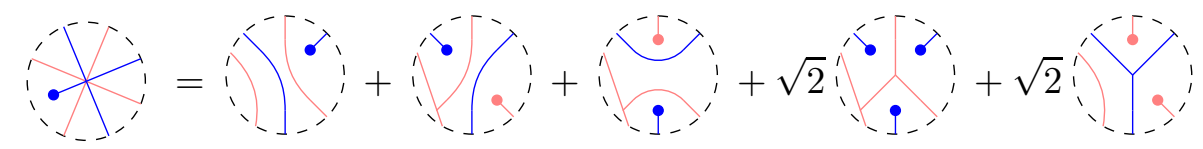

and one obtains the other relation by swapping red and blue. 
On the other hand if we take the "Cartan matrix" realization with

$$
\left\langle\alpha_{t}^{\vee}, \alpha_{s}\right\rangle=-2 \quad \text { and } \quad\left\langle\alpha_{s}^{\vee}, \alpha_{t}\right\rangle=-1
$$

(so that the non-simple positive roots are $\alpha_{s}+\alpha_{t}$ and $\alpha_{s}+2 \alpha_{t}$ ) then the relation is not stable under interchanging red and blue, and the Jones-Wenzl relations are
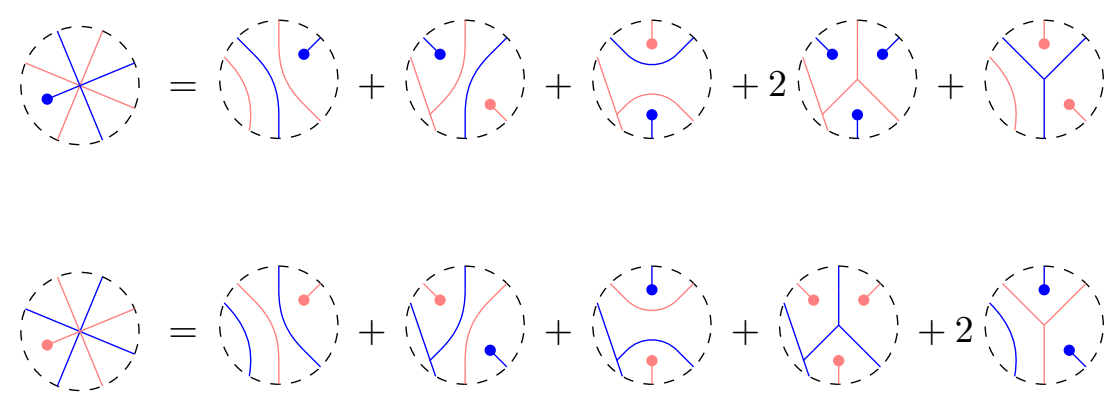

Remark 2.2. A useful mnemonic to remember the placement of the coefficients in the Jones-Wenzl relation is given by the relation

$$
\text { : }
$$

where $\pi_{s t}$ denotes the product of all positive roots in the root subsystem corresponding to $s, t$. The reader can convince themselves that each JonesWenzl relation given above implies that (2.1) holds. With some practice, (2.1) can be used to remember the placement of the coefficients above.

\subsubsection{Zamolodchikov relations}

We will not repeat the definition of the Zamolodchikov relations here, and instead refer the reader to [6, $\S 1.4 .3]$. (See [7] for topological background).

2.8. All relations defining $\mathbb{S D}$ are homogenous for the grading on $S$-graphs defined in $\S 2.6$. It follows that the $\mathbb{S D}$ is enriched in graded $\mathbb{k}$-modules. Throughout it will be convenient to view $\mathbb{S D}$ as enriched in graded left $R$ - 
modules via

$$
f \cdot D=f D
$$

for an $S$-graph $D$ and homogenous $f \in R$.

Given a $S$-graph $D$ we denote by $\bar{D}$ the $S$-graph obtained by flipping the diagram vertically. This operation induces a contravariant equivalence (also denoted $G \mapsto \bar{G}$ on morphisms) of the monoidal category $\mathbb{S D}$.

2.9. For any $x \in W$, let $\mathbb{S} \mathcal{D} \nless x$ be the quotient category of $\mathbb{S} \mathcal{D}$ by the ideal $\mathbb{S D}_{<x}$ of $\mathbb{S D}$ generated by all the morphisms which factor through $\underline{y}$, where $\underline{y}$ is a reduced expression for some $y<x$. Let $\operatorname{Hom} \nless x(-,-)$ denote morphisms in $\mathbb{S D}^{\nless x}$. The image of any reduced expression for $x$ in $\mathbb{S D}^{\nless x}$ yields an object defined up to canonical isomorphism, independent of the choice of reduced expression (see [6, 6.5]). We denote this object $x$. We have End $\nless x(\mathrm{x})=\mathrm{R}$.

By [6, Theorem 1.1], $\operatorname{Hom}^{\nless \mathrm{x}}(\underline{\mathrm{w}}, \mathrm{x})$ and $\operatorname{Hom} \nless \mathrm{x}(\mathrm{x}, \underline{\mathrm{w}})$ are free (graded) $R$ modules with graded basis given by the (images of the) light leaves $L L_{\underline{w}, \underline{e}}$ and $\overline{L L}_{\underline{w}, \underline{e}}$ respectively, where $\underline{e}$ runs over the 01-sequences with $\left(\underline{w}^{\underline{e}}\right) \bullet=x$. The light leaves are constructed in $[6, \S 6.1]$. For any $\underline{w} \in \operatorname{Ex}(\mathrm{S})$ the intersection form for $\underline{w}$ at $x$ is the $R$-bilinear pairing of graded free $R$-modules

$$
I_{x, \underline{w}}: \operatorname{Hom}^{\nless \mathrm{x}}(\mathrm{x}, \underline{\mathrm{w}}) \times \operatorname{Hom}^{\nless \mathrm{x}}(\underline{\mathrm{w}}, \mathrm{x}) \rightarrow \operatorname{End}^{\nless \mathrm{x}}(\mathrm{x})=\mathrm{R} .
$$

2.10. We give two examples of intersection forms.

First, assume that $W$ is a dihedral group with simple reflections $s$ and $t$. Let $\underline{w}=s t s, x=s$. There are two subexpressions for $x$ : $\mathbf{e}^{1}=(U 1, U 0, D 0)$ (defect 0$)$ and $\mathbf{e}^{2}=(U 0, U 0, U 1)$ (defect 2). The corresponding light leaves morphisms are

$$
l_{1}=\oint \quad \text { and } \quad l_{2}=\emptyset .
$$

The intersection form is given by the matrix:

$$
\left(l_{i} \circ \overline{l_{j}}\right)_{i, j \in\{1,2\}}=\left(\begin{array}{cc}
\left\langle\alpha_{t}, \alpha_{s}^{\vee}\right\rangle & \alpha_{t} \\
\alpha_{t} & \alpha_{s} \alpha_{t}
\end{array}\right)
$$


For example, the upper left entry follows from the following calculation:

$$
\gamma=\alpha_{t}=\partial_{s}\left(\alpha_{t}\right)+s\left(\alpha_{t}\right)
$$

(we use the barbell, nil Hecke and needle relations, as well as $\partial_{s}\left(\alpha_{t}\right)=$ $\left.\left\langle\alpha_{t}, \alpha_{s}^{\vee}\right\rangle\right)$. This example shows the existence of torsion in the intersection cohomology of the Schubert variety indexed by sts if $\left\langle\alpha_{t}, \alpha_{s}^{\vee}\right\rangle<-1$ (as happens in $B_{2}, G_{2}$ and $\widetilde{A_{1}}$ ).

For the second example, assume that $W$ is of type $D_{4}$ with generators $s, t, u, v$ such that $s, u$ and $v$ commute. Let $\underline{w}=v u v t s u v, x=s u v$. We only give the part of the intersection form corresponding to light leaves morphisms of degree 0 . Then there are three subexpressions of defect 0 . These subexpressions, and the corresponding light leaves maps are the following:

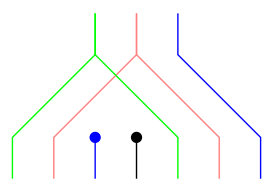

U1 U1 U0 U0 Do Do U1.

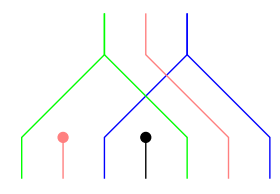

U1 U0 U1 U0 Do U1 Do

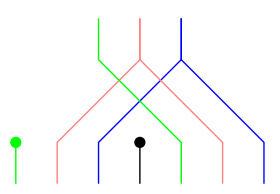

U0 U1 U1 U0 U1 Do Do

We leave it to the reader to pair these morphisms and obtain the intersection form

$$
\left(\begin{array}{ccc}
0 & -1 & -1 \\
-1 & 0 & -1 \\
-1 & -1 & 0
\end{array}\right)
$$

Note that the determinant of this matrix is -2 . This example of 2-torsion in the $D_{4}$ flag variety was discovered by Braden [21, A.18].

The goal of this paper is to show that light leaves morphisms corresponding to sequences without $D 1$ (like those above) are canonical and to give a formula for the corresponding entry in the intersection form in terms of the nil Hecke ring.

2.11. Let $\mathbb{S D}_{\oplus}$ denote the additive graded envelope of $\mathbb{S} \mathcal{D}$. That is, objects of $\mathbb{S D}_{\oplus}$ are formal direct sums of shifts of the objects $\underline{w}$ in $\mathbb{S D}$, with obvious morphisms. We denote by $\mathbb{S D}_{\oplus, 0}$ the subcategory of $\mathbb{S D}_{\oplus}$ consisting only of 
degree zero morphisms, and by $\operatorname{Kar}(\mathbb{S} \mathcal{D})$ the Karoubian envelope of $\mathbb{S D}_{\oplus, 0}$. Then $\operatorname{Kar}(\mathbb{S} \mathcal{D})$ is a $\mathbb{k}$-linear category with grading shift functor [1].

Under the assumptions of $\S 2.5$, the main theorems of [6] give a basis for hom spaces in $\mathbb{S} \mathcal{D}$ and deduce a classification of the indecomposable objects up to isomorphism and shift: for any $x$ and reduced expression $\underline{x}$ for $x, \underline{x}$ has a unique summand $b_{x}$ which is not a summand up to shift of $\underline{y}$ for any $y<x$, and any indecomposable object in $\operatorname{Kar}(\mathbb{S D})$ is isomorphic to $b_{x}[m]$ for some $x \in W$ and $m \in \mathbb{Z}$.

From this one deduces an isomorphism of $\mathbb{Z}\left[v, v^{-1}\right]$-algebras

$$
\varepsilon: \mathcal{H} \stackrel{\sim}{\rightarrow}[\operatorname{Kar}(\mathbb{S} \mathcal{D})] \quad \underline{\mathrm{H}}_{\underline{\mathrm{w}}} \mapsto \underline{\mathrm{w}} .
$$

where $[\operatorname{Kar}(\mathbb{S} \mathcal{D})]$ denotes the split Grothendieck group of $\operatorname{Kar}(\mathbb{S} \mathcal{D})$. For any $\underline{w} \in \operatorname{Ex}(\mathrm{S})$ one has in $[\operatorname{Kar}(\mathbb{S D})]$

$$
[\underline{w}]=\sum_{x \in W} m_{x}\left[b_{x}\right]
$$

where $m_{x}$ denotes the graded rank of the intersection form $I_{x, \underline{w}}$. This explains the central importance of the intersection forms.

In [5] it is proved (using Soergel bimodules) that if $\mathbb{k}$ is a field of characteristic 0 then $\varepsilon$ sends the Kazhdan-Lusztig basis element $\underline{H}_{w}$ to the class of $b_{w}$.

2.12. Though it will not be used in this paper, we briefly explain the connection to Soergel bimodules. Let us assume that $\mathbb{k}$ is a field of characteristic $\neq 2$.

For any $s \in S$, we denote by $R^{s} \subset R$ the invariant subring and

$$
B_{s}=R \otimes_{R^{s}} R[1]
$$

a graded $R$-bimodule. For any $\underline{w}=\left(s_{1}, \ldots, s_{m}\right) \in \operatorname{Ex}(\mathrm{S})$, we define the corresponding Bott-Samelson bimodule to be

$$
B_{\underline{w}}:=B_{s_{1}} \otimes_{R} B_{s_{2}} \otimes_{R} \cdots \otimes_{R} B_{s_{m}}
$$

Let $\mathbb{S B i m}$ be the category of Soergel bimodules, that is, the Karoubi envelope of $B_{\underline{w}}$ for all $\underline{w} \in \operatorname{Ex}(\mathrm{S})$ inside the category of graded $R$-bimodules. 
By [19, Satz] $]^{\|}$for any $w \in W$, there exists a unique (up to isomorphism) indecomposable $R$-bimodule $B_{w}$ which occurs as a direct summand of $B_{\underline{w}}$ for any reduced expression $\underline{w}$ with $\underline{w}_{\bullet}=w$, and does not occur as a direct summand of $B_{\underline{x}}$ for any $\underline{x}$ with $w \not \leq \underline{x}_{*}$. The set $\left\{B_{w}\right\}_{w \in W}$ is a complete set of indecomposable Soergel bimodules (up to isomorphism and degree shift).

In 6] a functor $\mathcal{F}: \mathbb{S D} \rightarrow \mathbb{S B i m}$ is constructed and it is proved that $\mathcal{F}$ induces an equivalence of monoidal categories $\operatorname{Kar}(\mathbb{S} \mathcal{D}) \stackrel{\sim}{\rightarrow} \mathbb{S B i m}$. In particular, $\mathcal{F}$ maps $b_{w}$ to $B_{w}$, for any $w \in W$.

\section{Gobbling Morphisms}

3.1. In general light leaves morphisms are not canonical, and this causes many complications. For example it seems difficult to make all entries in intersection forms canonical. In this section we introduce gobbling morphisms, which are certain canonical morphisms between Soergel bimodules.

The aim of this section is to prove:

Proposition 3.1. Let $\underline{w}=u_{1} \ldots u_{n}$ be an expression. Any two morphisms $\underline{w} \rightarrow \underline{w}_{*}$ in $\mathbb{S D} \nless \underline{w}_{*}$ given by diagrams consisting only of $2 m_{\text {st }}$-valent vertices and $\ell(\underline{w})-\ell\left(\underline{w}_{*}\right)$ trivalent vertices are equal.

Definition 3.2. We call the morphism $\underline{w} \rightarrow \underline{w}_{*}$ in $\mathbb{S D}^{\nless \underline{w}_{*}}$ whose unicity is given by the previous proposition the gobbling morphism and denote it by $G_{\underline{w}}$.

Remark 3.3. One has $\operatorname{deg} G_{\underline{w}}=-\left(\sharp\right.$ of trivalent vertices in $\left.G_{\underline{w}}\right)=\ell\left(\underline{w}_{*}\right)-$ $\ell(\underline{w})$.

For $\underline{w}$ in the proposition we can consider the subexpression $\underline{e}:=e_{1} \ldots e_{n}$ defined inductively by the recipe

$$
e_{i}:= \begin{cases}1 & \text { if } w_{i-1} s_{i}>w_{i-1} \\ 0 & \text { otherwise }\end{cases}
$$

where $w_{i}:=\left(\underline{w}_{\leq i}^{\underline{e}} \leq i\right)$. Obviously $\left(\underline{w}^{\underline{e}}\right)_{\bullet}=\underline{w}_{*}$. Also note that the decoration of $\underline{e}$ consists entirely of U1's and D0's. Hence (any choice of) the light leaf

\footnotetext{
${ }^{1}$ under some mild assumptions on the realization $\mathfrak{h}$ (which we ignore here).
} 
morphism $L_{\underline{w}, \underline{e}}$ consists only of $2 m_{s t}$-valent vertices and exactly $\ell(\underline{w})-\ell\left(\underline{w}_{*}\right)$ trivalent vertices. In particular $L_{\underline{w}, \underline{e}}$ satisfies the conditions of Proposition 3.1. 3.1 as:

Let $l_{\underline{w}}$ denote the image of $L_{\underline{w}, \underline{e}}$ in $D \nless \underline{w}_{*}$. We can restate Proposition

Proposition 3.4. Any morphism $\underline{w} \rightarrow \underline{w}_{*}$ in $\mathbb{S} \mathcal{D} \nless \underline{w}_{*}$ satisfying the conditions of Proposition 3.1 is equal to $l_{\underline{w}}$.

This is the version we will prove. We start with some preparatory lemmata. The first lemma shows that the space in which the gobbling morphism(s) live is free of rank one, and that this degree is the minimal non-zero degree:

Lemma 3.5. Hom $\underline{\mathrm{w}}_{*}\left(\underline{\mathrm{w}}, \underline{\mathrm{w}}_{*}\right)$ is zero in degrees $<\ell\left(\underline{w}_{*}\right)-\ell(\underline{w})$ and the degree $\ell\left(\underline{w}_{*}\right)-\ell(\underline{w})$ part of $\operatorname{Hom} \nless \underline{w}_{*}\left(\underline{\mathrm{w}}, \underline{\mathrm{w}}_{*}\right)$ is of rank 1 .

Proof. We know that $\operatorname{Hom}^{\nless \underline{\mathrm{w}}_{*}}\left(\underline{\mathrm{w}}_{\mathrm{w}} \underline{\mathrm{w}}_{*}\right)$ has a basis given by light leaf morphisms corresponding to 01-sequences $\underline{e}^{\prime}$ with $\left(\underline{w}^{e^{\prime}}\right) \bullet=\underline{w}_{*}$. Notice that $\left(\underline{w}^{e^{\prime}}\right) \cdot=\underline{w}_{*}$ implies that the number of 0 's in $\underline{e}^{\prime}$ is less than or equal to $\ell(\underline{w})-\ell\left(\underline{w}_{*}\right)$. Hence $\operatorname{df}\left(\underline{\mathrm{e}}^{\prime}\right) \geq \ell\left(\underline{\mathrm{w}}_{*}\right)-\ell(\underline{\mathrm{w}})$. If the equality holds, then there are exactly $\ell\left(\underline{w}_{*}\right) U 1$ 's and exactly $\ell(\underline{w})-\ell\left(\underline{w}_{*}\right) D 0$ 's in $\underline{e}^{\prime}$. In this case, $\underline{e}^{\prime}$ equals $\underline{e}$ defined above.

Lemma 3.6. Suppose that $f: \underline{w} \rightarrow \underline{w}_{*}$ is a morphism in $\mathbb{S D} \nless \underline{w}_{*}$ of degree $\ell\left(\underline{w}_{*}\right)-\ell(\underline{w})$. Let $\underline{e}$ be a subsequence of $\underline{w}$ such that $\underline{e}$ has at least one D0 or D1. Fix a choice of light leaf morphism $L L_{\underline{w}, \underline{e}}: \underline{w} \rightarrow\left(\underline{w}^{\underline{e}}\right)$. in $\mathbb{S D}$. In $\mathbb{S D} \nless \underline{w}_{*}$ we have:

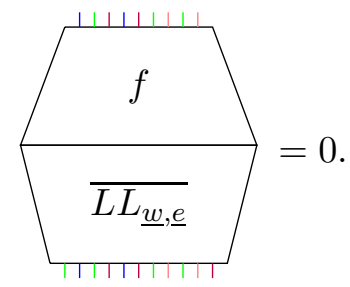

Proof. When we precompose $f$ with a $2 m$-valent vertex we obtain a morphism of the same form. If we precompose $f$ with a dot map $g: \underline{w}^{\prime} \rightarrow \underline{w} \stackrel{f}{\rightarrow} \underline{w}_{*}$ then there are two cases: 
(1) if $\underline{w}_{*}^{\prime}=\underline{w}_{*}$ then our new morphism $g$ is of the same form as $f$;

(2) if $\underline{w}_{*}^{\prime} \neq \underline{w}_{*}$ then $\underline{w}_{*}^{\prime}<\underline{w}_{*}$ and our new morphism $g$ is zero in $\mathbb{S} \mathcal{D}^{\nless \underline{w}_{*}}$.

Hence by our assumptions on $\underline{e}$ we can assume that we precompose $f$ with a trivalent vertex 1$\}^{-}$or cup $1 \underbrace{}_{-1}$ (which factors through a trivalent vertex). Then the fact that these morphisms are 0 follows from the previous lemma by degree considerations.

Let us say that an $S$-graph ends in a pitchfork if it is equivalent modulo isotopy and the Frobenius relations to an $S$-graph of the form

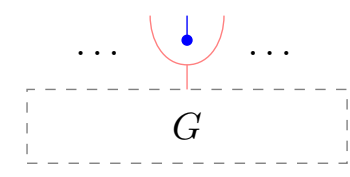

for some $S$-graph $G$. For example, the morphisms

and

both end in pitchforks. The following lemma is proved in the same way as the previous lemma.

Lemma 3.7. Suppose that $f: \underline{w} \rightarrow \underline{w}_{*}$ is a morphism in $\mathbb{S} \mathcal{D} \nless \underline{w}_{*}$ of degree $\ell\left(\underline{w}_{*}\right)-\ell(\underline{w})$. Then $f$ is "killed by all pitchforks": if $u_{i} u_{i+1} u_{i+2}=$ sts for some $i$ and simple reflections $s, t \in S$ then:

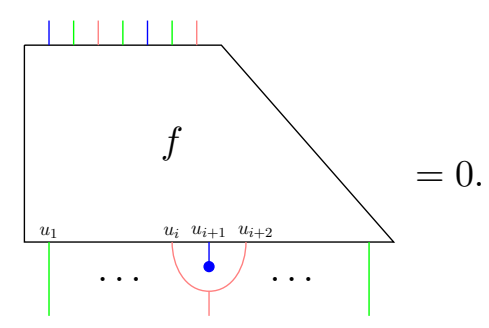

This lemma has as a consequence that that adding the "square" of a $2 m_{s t}$-valent vertex does not change a gobbling morphism:

Corollary 3.8. Suppose that $f: \underline{w} \rightarrow \underline{w}_{*}$ is a morphism in $\mathbb{S D}^{\nless \underline{w}_{*}}$ of degree 
$\ell\left(\underline{w}_{*}\right)-\ell(\underline{w})$. Let $i$ be such that $u_{i} u_{i+1} \ldots u_{i+m_{s t}-1}=$ sts $\ldots$ Then

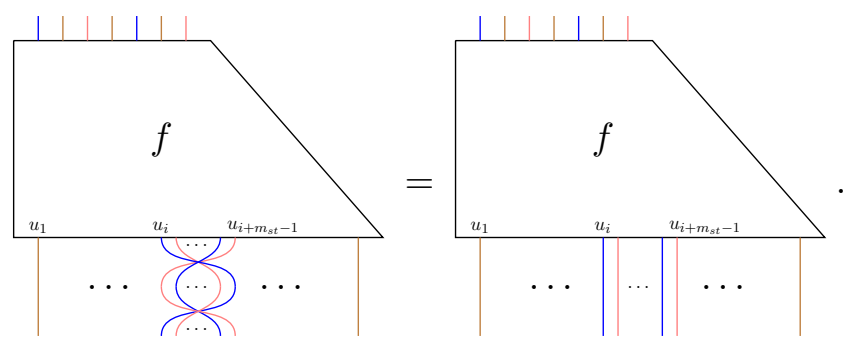

Proof. As explained in [3, Claim 6.7], two-color associativity $\S 2.7 .6$ and the Jones-Wenzl relation $§ 2.7 .7$ imply that we can write

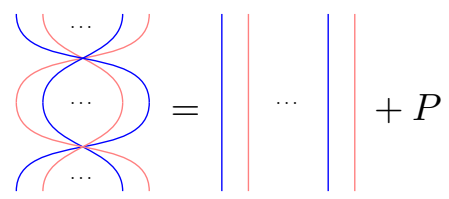

where all terms in $P$ end in pitchforks. Now the result follows from Lemma 3.7.

Proposition 3.9. If $\underline{e}$ is defined as in (3.1), then the images of any two choices of light leaves morphisms $L_{\underline{w}, \underline{e}}$ and $L_{\underline{w}, \underline{e}}^{\prime}$ in $\mathbb{S} \mathcal{D} \nless \underline{w}_{*}$ agree.

In the proof of the proposition we will need the notion of a rex move from [6, $\S 4.2]:$ a rex move $\beta: \underline{x} \rightarrow \underline{x}^{\prime}$ is a sequence of reduced expressions $\underline{x}=\underline{x}_{0} \rightarrow \underline{x}_{1} \rightarrow \ldots \underline{x}_{m}=\underline{x}^{\prime}$ such that each move $\underline{x}_{i} \rightarrow \underline{x}_{i+1}$ involves the application of a single braid relation $\left(s t \ldots \rightarrow t s \ldots\right.$ ( $m_{s t}$-factors) for $m_{s t} \geq$ 2 ). Any two reduced expressions can be linked via rex moves (a theorem of Matsumoto, see e.g. [15, Theorem 1.9]). Rex moves give morphisms in $\mathbb{S} \mathcal{D}$ by composing the corresponding $2 m_{s t}$-valent vertices (see [6, $\left.\S 6.1\right]$ ).

Proof. We prove the proposition by induction on the defect $\operatorname{df}(\underline{\mathrm{e}})$ of $\underline{e}$. If $\mathrm{df}(\underline{\mathrm{e}})=0$ then a choice of light leaf morphism is simply a choice of reduced expression $\underline{x}$ for $\underline{w}$. together with a rex move $\underline{w} \rightarrow \underline{x}$. The corollary follows in this case because the difference between any two rex moves lies in $\mathbb{S} \mathcal{D}_{<\underline{w}_{*}}$ (a consequence of Elias' Jones-Wenzl relation and the Zamolodchikov relations, see [6, Lemma 7.4]). 
We now assume that $\operatorname{df}(\underline{\mathrm{e}}) \geq 1$ and let $i+1$ denote the position of the first $D 0$ in $\underline{e}$. We can draw two choices of light leaves morphisms as
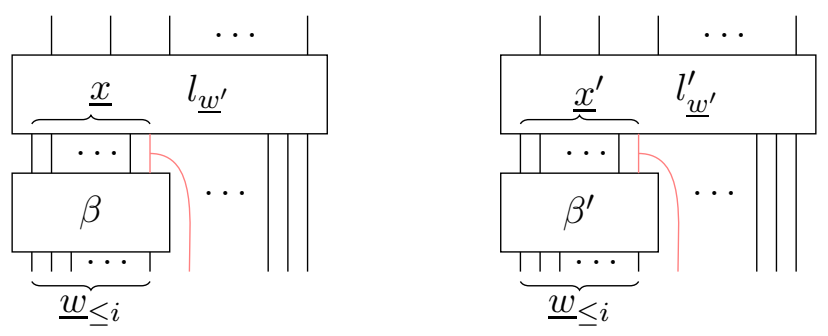

where $\underline{x}, \underline{x}^{\prime}$ are reduced expressions for $\left(\underline{w}_{\leq i}\right)$ • both ending in $s_{i+1}$ (coloured red in the above diagrams). By applying the previous corollary we can replace $\beta^{\prime}$ by a rex move of the form:

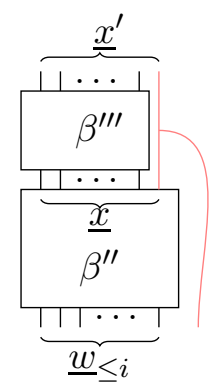

Now we can slide $\beta^{\prime \prime \prime}$ into $l_{\underline{w^{\prime}}}^{\prime}$ in the right hand diagram above. Hence we can assume $\underline{x}=\underline{x}^{\prime}$ in the above diagram. Now we can apply induction to conclude that $l_{\underline{w^{\prime}}}=l_{\underline{w^{\prime}}}^{\prime}$. Thus we can assume that the above two diagrams are identical except in the boxes corresponding to $\beta$ and $\beta^{\prime}$. Now the result follows from Lemma 3.6 and the lemma below.

Lemma 3.10. Let $\beta_{1}, \beta_{2}$ be two rex moves from $\underline{w}_{1} \rightarrow \underline{w}_{2}$, where $\underline{w}_{1}$ and $\underline{w}_{2}$ are reduced expressions. Then we can write

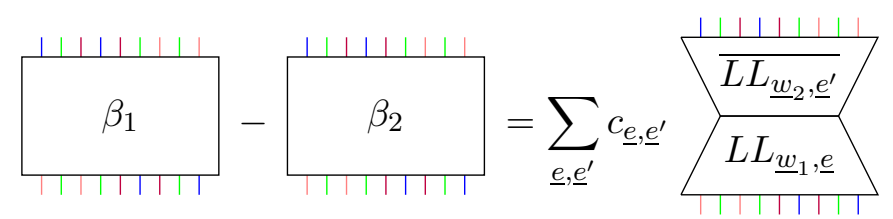

for homogenous $c_{\underline{e}, \underline{e}^{\prime}} \in R$ and where $L L_{\underline{w}_{1}, \underline{e}}, L L_{\underline{w}_{2}, \underline{e}^{\prime}}$ are light leaf morphisms corresponding to subsequences $\underline{\underline{e}}, \underline{e}^{\prime}$ having at least one D1 or D0. 
Proof. We know that the left hand side vanishes in $\mathbb{S D}^{\nless} \underline{w}_{1} \bullet$ and hence all terms on the right hand side factor through some $\underline{y}$ with $\underline{y} \bullet<\left(\underline{w}_{1}\right)_{\bullet}$. Assume for contradiction that there is a term on the right hand side with $c_{\underline{e}, \underline{e}} \neq 0$, where $\underline{e}$ is a subsequence of $\underline{w}_{1}$ without $D 1$ or $D 0$. In particular, $\operatorname{df}(\underline{\mathrm{e}})=$ $\ell\left(\underline{\mathrm{w}}_{1}\right)-\ell\left(\left(\underline{\mathrm{w}}_{1} \underline{\mathrm{e}}\right) \bullet\right)$. For degree reasons $\operatorname{df}\left(\underline{\mathrm{e}}^{\prime}\right)+\operatorname{deg}\left(\mathrm{c}_{\underline{\mathrm{e}}, \underline{\mathrm{e}}^{\prime}}\right)=\ell\left(\left(\underline{\mathrm{w}}_{1} \underline{\mathrm{e}}\right) \bullet\right)-\ell\left(\underline{\mathrm{w}}_{1}\right)$. However $\operatorname{df}\left(\underline{\mathrm{e}}^{\prime}\right) \geq \ell\left(\left(\underline{\mathrm{w}}_{1} \underline{\mathrm{e}}\right) \bullet\right)-\ell\left(\underline{\mathrm{w}}_{1}\right)$ and hence $\operatorname{deg}\left(c_{\underline{e}, \underline{e}^{\prime}}\right)=0$ and $\operatorname{df}\left(\underline{\mathrm{e}}^{\prime}\right)=$ $\ell\left(\left(\underline{\mathrm{w}}_{1}\right)\right.$ is impossible as $\underline{w}_{2}$ is reduced.

\subsection{Proof of Proposition 3.4 in rank 2}

Assume that $W$ is of rank 2, i.e. a dihedral group. We denote the simple reflections of $W$ by $s$ and $t$ and denote the order of $s t$ (possibly $\infty$ ) by $m$. The longest element of $W$ is $w_{0}$ (if it exists).

If $m=\infty$ then the proposition is easy: only trivalent vertices can occur, and the proposition follows from the fact that any two morphisms

$$
s s \ldots s \rightarrow s \quad(k \text { factors on the left })
$$

consisting of exactly $k$ trivalent vertices and no dots are equal (a consequence of the Frobenius associativity relation $§ 2.7 .2)$.

From now on we will assume that $m$ is finite. If $\underline{w}_{*} \neq w_{0}$ then the result follows similarly to the case $m=\infty$ above. So we may assume $\underline{w}_{*}=w_{0}$.

Let us denote by $l_{\underline{w}}$ the light leaves map $\underline{w} \rightarrow \underline{w}_{*}$ as above. In this section (i.e. until $\S 3.3$ ) we always regard $l_{\underline{w}}$ as a morphism to $b_{w_{0}}$. That is we always compose with some number $\geq 1$ of iterates of the $2_{m_{s t}}$-valent vertex. 
We can depict $l_{\underline{w}}$ schematically as follows (we depict the case $m=4$ ):

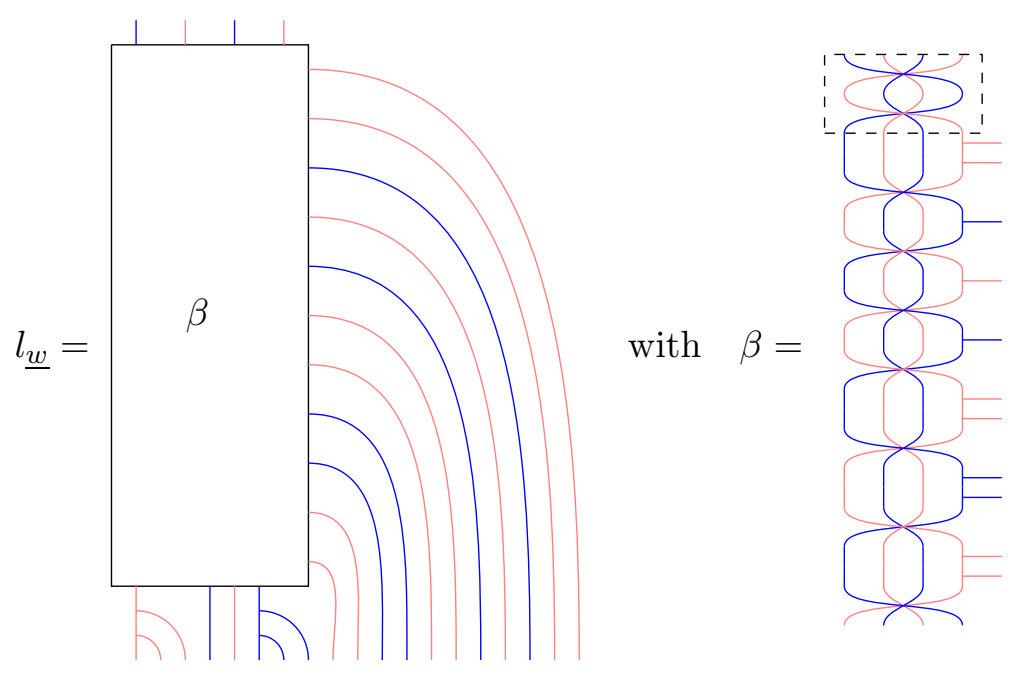

(The dashed region in $\beta$ denotes any $k \geq 1$ compositions of $2 m$-valent vertices which will be implicit (and not displayed) in all diagrams until §3.3.)

Lemma 3.11. Let $\underline{w} \in \operatorname{Ex}(\mathrm{S})$ with $\underline{w}_{*}=w_{0}$. In $\operatorname{Hom}\left(\underline{\mathrm{w}}, \mathrm{b}_{\mathrm{w}_{0}}\right)$ we have:

(1)

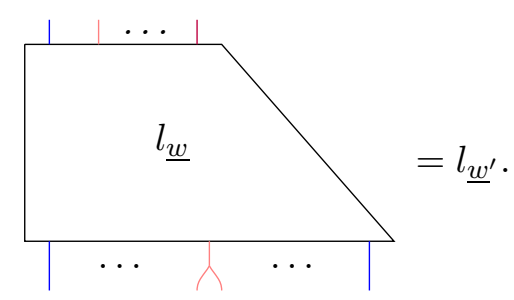

(2) If $\underline{w}$ is of the form $\underline{w}=u_{1} \ldots u_{n}$ with $u_{i} u_{i+1} \ldots u_{i+m-1}=$ sts $\ldots$ ( $m$ times). Then, in $\operatorname{Hom}\left(\underline{\mathrm{w}}, \mathrm{b}_{\mathrm{w}_{0}}\right)$ :

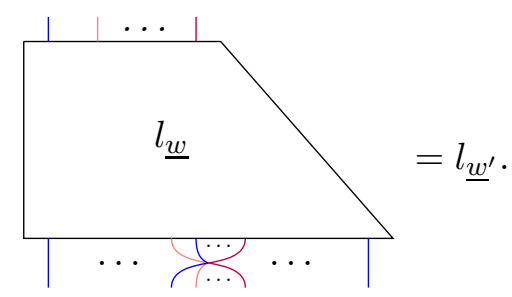

(In (1) and (2) the definition of $\underline{w}^{\prime}$ should be clear from the diagrams.) 
This lemma is clearly equivalent to Proposition 3.4. (The apparently missing case of composing with a splitting trivalent vertex $\left.\begin{array}{ll}i_{1} \\ \vdots\end{array}\right]_{-1}^{\prime}$ does not satisfy the conditions of Proposition 3.1.) Also, part (1) of the lemma follows directly from the definitions and the Frobenius associativity relation $§ 2.7 .2$. After examining the above picture of $l_{\underline{w}}$ it is not difficult to see that $(2)$ is implied by the following two claims:

Claim 3.12. We have:

$(m$ even $)$

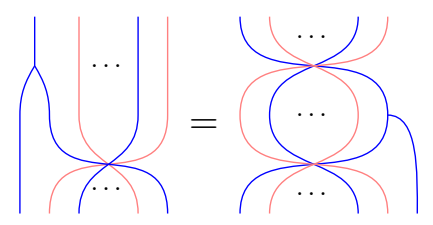

$(m$ odd $)$

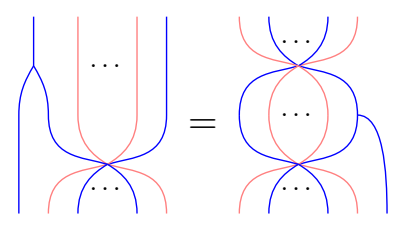

Claim 3.13. Suppose $k \leq m$ and $\underline{w}$ is of the form

$$
\underline{w}=s t s \ldots \quad(k+m \text {-factors })
$$

Then in $\operatorname{Hom}\left(\underline{\mathrm{w}}^{\prime}, \mathrm{b}_{\mathrm{w}_{0}}\right)$ we have:
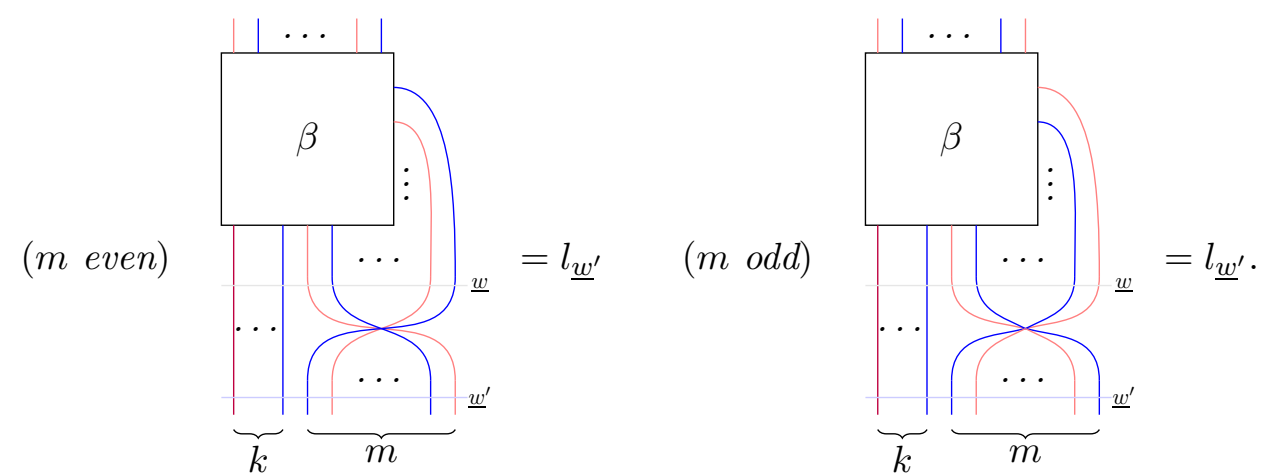

(In both cases the purple line indicates a line that is either blue or red, depending on the parity of $k$.)

The first claim is just a restatement of the two-colour associativity relation $\S 2.7 .6$. It remains to prove Claim 3.13 .

We prove Claim 3.13 by induction on $k$. For $k=0$ there is nothing to prove. The case $k=1$ is again a restatement of two-colour associativity relation $\S 2.7 .6$. For concreteness we assume that $m$ is even. It is not difficult to check that the same argument works for $m$ odd. 
Assume first that $k$ is even. Then we can write

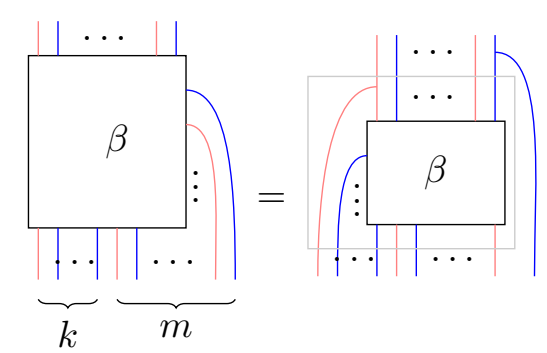

(we use induction and Proposition 3.4 to rewrite the term on the right hand side enclosed in the gray box as a light leaves morphism). Hence the term in the claim is equal to:

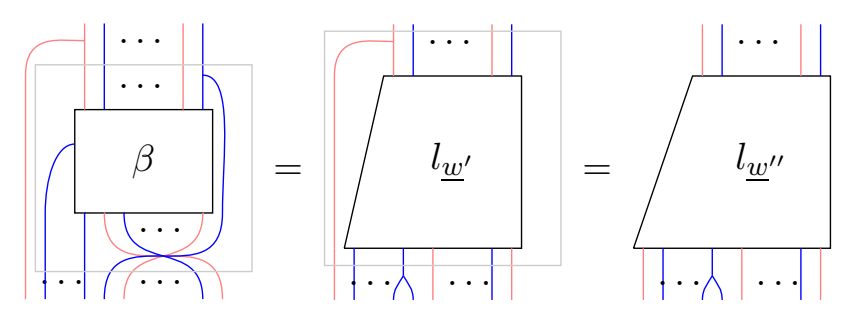

(at each step we apply induction to the term in the gray box). Now we are done by part (1) of Lemma 3.11.

Now assume that $k$ is odd. Then we have

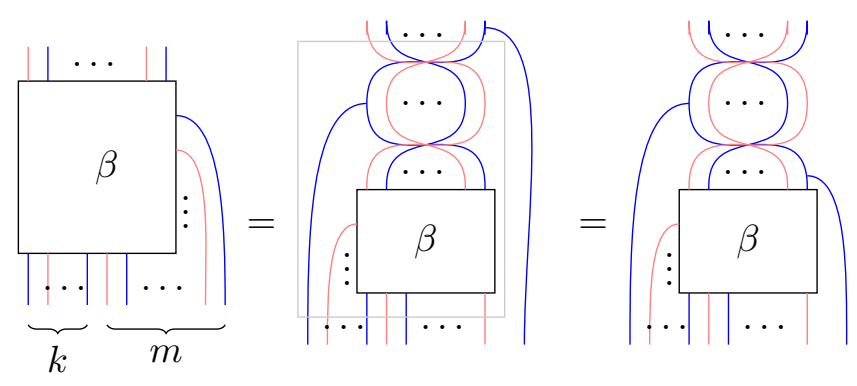

(again for the first step we apply induction and Proposition 3.4 to simplify 
the term in the gray box). For the second equality we have used the relation

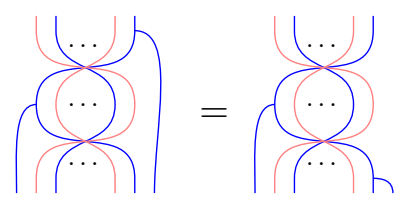

which follows by induction (or can be checked direction from two colour associativity). Now the argument proceeds as in the case of $k$ even.

\subsection{Proof of Proposition 3.4 in general}

Now take $W$ any Coxeter system. As in the dihedral case it is enough to establish the following claim:

Lemma 3.14. Let $\underline{w} \in \operatorname{Ex}(\mathrm{S})$ with $\underline{w}_{*}=x$. In $\mathbb{S D}^{\nless x}$ we have:

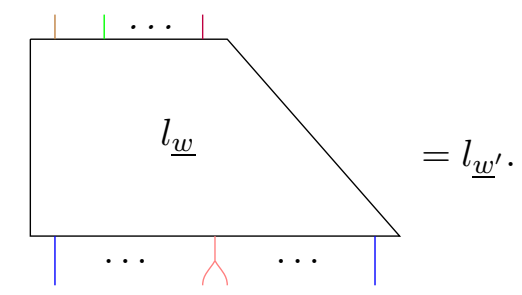

(2) If $\underline{w}$ is of the form $\underline{w}=u_{1} \ldots u_{n}$ with $u_{i} u_{i+1} \ldots u_{i+m-1}=$ sts $\ldots$ ( $m$ times). Then

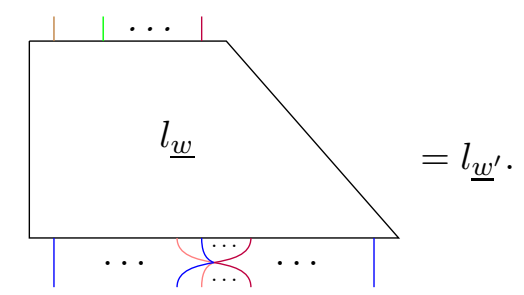

(In (1) and (2) the definition of $\underline{w}^{\prime}$ should be clear from the diagrams.)

As in the dihedral case (1) is immediate from the definitions. It remains 
to prove (2). We depict $l_{\underline{w}}$ schematically as follows:

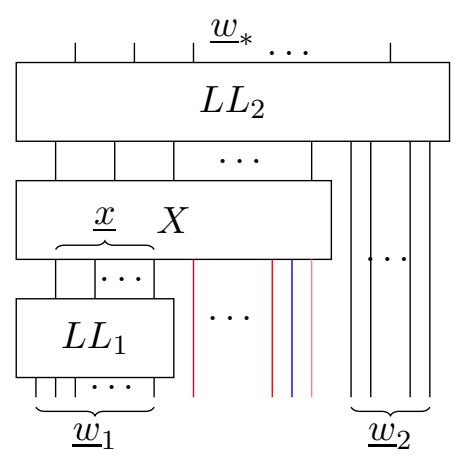

(Here $\underline{w}_{1}=u_{1} \ldots u_{i-1}, \underline{w}_{2}=u_{i+m} \ldots u_{n}$ and $\underline{x}$ is a reduced expression for $\left(\underline{w}_{\leq i-1}^{\underline{e}} \leq i-1\right)$. $)$ Let us write $\left(\underline{w}_{\leq i-1}^{\underline{e} \leq i-1}\right)$. as $x^{\prime} v$ where $x^{\prime}$ is minimal in $x^{\prime}\langle s, t\rangle$ and $v \in\langle s, t\rangle$. By Corollary 3.9 we can assume that $\underline{x}$ is of the form $\underline{x^{\prime}} \underline{v}$ where $\underline{x}^{\prime}(\operatorname{resp} . \underline{v})$ is a reduced expression for $x^{\prime}$ (resp. $v$ ).

Moreover, by the construction of light leaves we can assume that $X$ has the form:

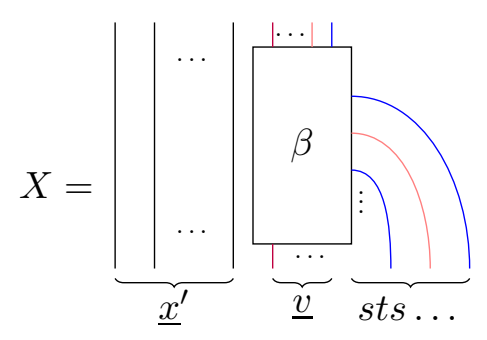

Now the claim reduces to a rank 2 calculation, which we have covered in the previous section.

\section{Properties of Gobbling Morphisms}

The following important property of gobbling morphisms explains their name:

Proposition 4.1. Let $\underline{w}=u_{1} \ldots u_{n}$. Fix $1 \leq i \leq n$ and set $\underline{w}^{\prime}:=$ 
$u_{1} \ldots u_{i-1} u_{i+1} \ldots u_{n}$. We have

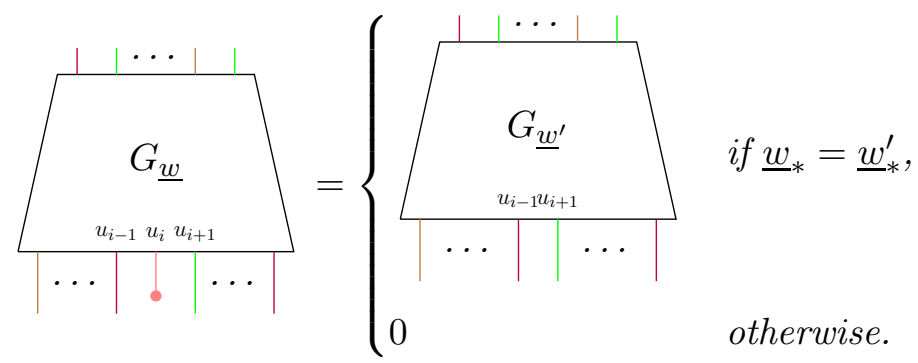

In the proof we will need the following lemma:

Lemma 4.2. The following relations hold. In each case $P$ is a linear combination of diagrams, all of which end in pitchforks:

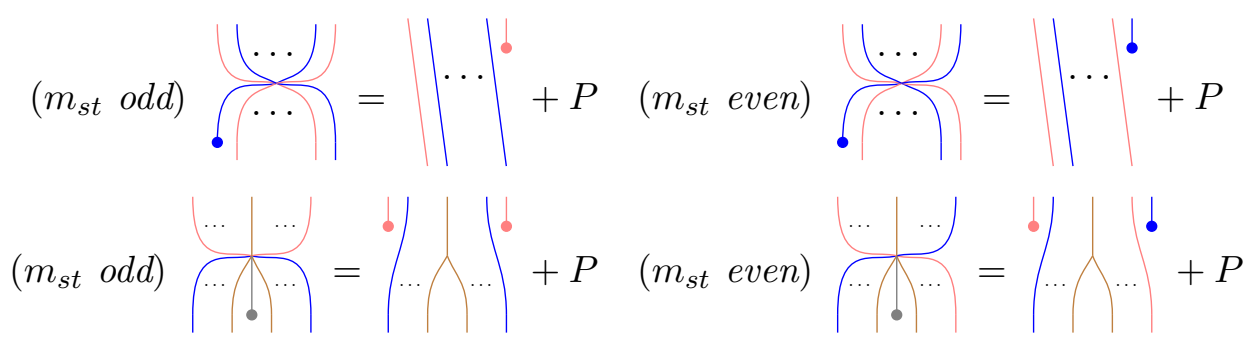

(In the second pair of relations the brown and gray lines can be chosen to be either red and blue, or blue and red.)

Proof. This is a consequence of Elias' Jones-Wenzl relation. (The only tricky point is to check that the coefficient of 1 in the right hand term is correct. The reader can verify directly that this is the case for $m_{s t}=2,3,4$ using the relations we have given.)

Proof. [Proof of Proposition 4.1] If $\underline{w}_{*}^{\prime} \neq \underline{w}_{*}$ then $\underline{w}_{*}^{\prime}<\underline{w}_{*}$ because $\underline{w}^{\prime}$ is a subexpression of $\underline{w}$ and the result is zero because we are working in $\mathbb{S} \mathcal{D} \nless \underline{w}_{*}$.

So now we assume that $\underline{w}_{*}^{\prime}=\underline{w}_{*}$. Let us induct on the number $N$ of $2 m_{s t}$-valent vertices which occur in $G_{\underline{w}}$. If $N=0$ then $G_{\underline{w}}$ consists only of trivalent vertices and the result is clear by the Frobenius unit relation $§ 2.7 .1$.

Now we examine what happens when we apply the relations to simplify the diagram on the left hand side of the proposition. If the dot first meets a trivalent vertex then the result is clear from the Frobenius unit relation 
$\S 2.7 .1$ (the source and target have the same star product because $s * s=s$ ). If the dot meets a $2 m$-valent vertex then we can apply Lemma 4.2 to expand it (and remove the $2 m$-valent vertex in question). By Lemma 3.7 we can ignore all the terms labelled $P$ in the lemma. Now we are done by induction (note that in each of the relations in Lemma 4.2 the expression corresponding to through strands on the bottom and top boundary of the pictured right hand term have the same $*$-product).

\section{Morphisms without D1's}

\subsection{The nil Hecke ring}

Denote by $Q$ the field of fractions of $R$.

Consider the smash product $Q_{W}:=Q * W$. That is, $Q * W$ is a free left $Q$-module with basis $\left\{\delta_{w} \mid w \in W\right\}$ and multiplication given by

$$
\left(f \delta_{x}\right)\left(g \delta_{y}\right)=f(x g) \delta_{x y}
$$

Inside $Q_{W}$ we consider the elements

$$
D_{s}:=\frac{1}{\alpha_{s}}\left(\delta_{\mathrm{id}}-\delta_{s}\right)=\left(\delta_{\mathrm{id}}+\delta_{s}\right) \frac{1}{\alpha_{s}} .
$$

The elements $D_{s}$ satisfy the relations:

$$
\begin{aligned}
D_{s}^{2} & =0 \\
D_{s} D_{t} \ldots & =D_{t} D_{s} \ldots \quad\left(m_{s t} \text {-factors on both sides }\right) \\
D_{s} f & =(s f) D_{s}+\partial_{s}(f) \quad \text { for all } f \in Q
\end{aligned}
$$

If $y \in W$ and $\underline{y}=s t \ldots u$ is a reduced expression then, by (5.2), we obtain well-defined elements

$$
D_{y}:=D_{s} D_{t} \ldots D_{u} \in Q_{W}
$$

The nil Hecke ring $N H$ is defined to be the $R$-subring of $Q * W$ generated by $\left\{D_{w} \mid w \in W\right\}$. (This is not the definition of Kostant-Kumar [13], but agrees with it for the realizations they consider.) As a left $R$-module $N H$ is free with basis $\left\{D_{w} \mid w \in W\right\}$. 
5.2. Given a word $\underline{w}=\left(s_{1}, \ldots, s_{m}\right)$ and a subexpression $\underline{e}$ we have a corresponding light leaves map $L L_{\underline{e}}: \underline{w} \rightarrow\left(\underline{w}^{\underline{e}}\right)$. in $\mathbb{S}^{\mathcal{D}} \nless \underline{w} \underline{\underline{e}}$. Now it is clear from construction that if the decoration of $\underline{e}$ has no D1's then $L L_{\underline{e}}$ has the form

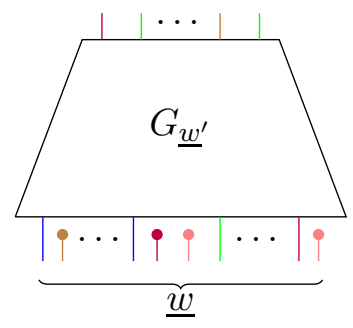

where $\underline{w}^{\prime}$ is the subword of $\underline{w}$ consisting of all $s_{i}$ such that $d_{i} e_{i} \neq U 0$ and $G_{\underline{w}^{\prime}}$ denotes the gobbling morphism associated to $\underline{w}^{\prime}$. In particular, the light leaves morphism $L L_{\underline{e}}$ is canonical in this case.

Consider two subexpressions $\underline{e^{1}}$ and $\underline{e^{2}}$ of $\underline{w}$ such that $\left(\underline{w} \frac{e^{1}}{}\right) \cdot\left(\underline{w} \frac{e^{2}}{}\right)$. Define an element of the nil Hecke ring as the product

$$
f\left(\underline{e^{1}}, \underline{e^{2}}\right)=f_{1} f_{2} \ldots f_{m}
$$

where

$$
f_{i}= \begin{cases}\alpha_{s_{i}} & \text { if } e_{i}^{1}=e_{i}^{2}=U 0 \\ 1 & \text { if exactly one of } e_{i}^{1} \text { and } e_{i}^{2} \text { is } U 0 \\ D_{s_{i}} & \text { otherwise }\end{cases}
$$

Finally, define $d\left(\underline{e^{1}}, \underline{e^{2}}\right)$ as the coefficient of $D_{\left(\underline{w} \underline{e^{1}}\right)}$ in $f\left(\underline{e^{1}}, \underline{e^{2}}\right)$. Hence $d\left(\underline{e^{1}}, \underline{e^{2}}\right) \in R$.

Theorem 5.1. If $\left(\underline{w} \underline{e^{1}}\right) \bullet=\left(\underline{w} \underline{e}^{\frac{e^{2}}{}}\right)$. and the decorations of $\underline{e^{1}}$ and $\underline{e^{2}}$ have no $D 1$, then

$$
\left\langle L L_{\underline{e^{1}}}, L L_{\underline{e^{2}}}\right\rangle=d\left(\underline{e^{1}}, \underline{e^{2}}\right) .
$$

Remark 5.2. Given a subexpression $\underline{e}$ of $\underline{w}$ let $J:=\left\{1 \leq i \leq m \mid e_{i}=1\right\}$ and write $J=\left\{i_{1}, \ldots, i_{r}\right\}$ with $i_{1}<i_{2}<\cdots<i_{r}$. Then $\underline{e}$ has no $D 1$ 's if and only if $\left(s_{i_{1}}, s_{i_{2}}, \ldots, s_{i_{r}}\right)$ is reduced. That is, $\underline{e}$ has no $D 1 \mathrm{~s}$ if the expression obtained from $\underline{w}^{\underline{e}}=\left(s_{1}^{e_{1}}, \ldots, s_{m}^{e_{m}}\right)$ by deleting all occurrences of id is reduced.

Proof. Set $x=\left(\underline{w} \underline{e^{1}}\right) \bullet=\left(\underline{w} \underline{e^{2}}\right)$. Let ${\underline{w^{\prime}}}^{1}$ be the expression obtained from $\underline{w}$ by deleting all the places $i$ with $d_{i} e_{i}^{1}=\bar{U} 0$ and ${\underline{w^{\prime}}}^{2}$ be the expression obtained 
from $\underline{w}$ by deleting all the places $i$ with $d_{i} e_{i}^{2}=U 0$. Then $x=\left(\underline{w}^{\prime 1}\right)_{*}=\left(\underline{w}^{\prime 2}\right)_{*}$. Therefore:

$$
L L_{\underline{w}, \underline{e^{1}}} \circ \overline{L L}_{\underline{w}, \underline{e^{2}}}=G_{\underline{w}^{1}} \circ K \circ \bar{G}_{{\underline{w^{\prime}}}^{2}}=\underbrace{\frac{\underline{w}^{\prime 2}}{\underline{w}}}_{{\underline{\underline{w^{\prime}}}}^{\prime 1}}
$$

The graph $K$ consists of vertical edges, vertical dotted edges and boxes. For example,

$$
K=\mid \downarrow\left\lfloor\alpha_{s_{4}}\left|\alpha_{s_{6}} \bullet\right| \downarrow \mid \alpha_{s_{11}} \uparrow\right.
$$

The graph $K$ is determined by $\underline{e^{1}}$ and $\underline{e^{2}}$ as follows. At the $i$ th place, we associate a box labelled by $\alpha_{s_{i}}$ if $d_{i} e_{i}^{1}=d_{i} e_{i}^{2}=U 0$, we associate a vertical dotted edge with label $s_{i}$ if exactly one of $d_{i} e_{i}^{1}$ and $d_{i} e_{i}^{2}$ is $U 0$ and associate a vertical edge colored by $s_{i}$ if neither $d_{i} e_{i}^{1}$ nor $d_{i} e_{i}^{2}$ is $U 0$ (here $* \in\{U 1, D 0\}$ ):

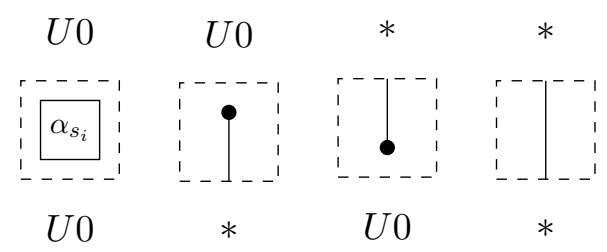

In the category $\mathbb{S} \mathcal{D}$, by using the nil Hecke relation we may write $K$ as an $R$-linear combination of graphs consisting of vertical edges, dotted edges and broken vertical edges. (See 2.7.5 for an example of this process and a broken edge.) More precisely, consider the following sets:

$$
\begin{gathered}
\underline{\varepsilon}:=\left\{i \mid\left(d_{i} e_{i}^{1}, d_{i} e_{i}^{2}\right) \neq(U 0, U 0)\right\}, \\
\underline{\varepsilon}_{\phi}:=\left\{i \mid \text { exactly one of } d_{i} e_{i}^{1}, d_{i} e_{i}^{2} \text { is } U 0\right\} .
\end{gathered}
$$

Thus $\underline{\varepsilon}$ indexes those positions in $K$ which do not correspond to boxes, and $\underline{\varepsilon}_{\phi}$ corresponds to those edges in $K$ which carry one dot.

For any $\underline{\gamma}$ with $\underline{\varepsilon}_{\phi} \subset \underline{\gamma} \subset \underline{\varepsilon}$, let $K_{\underline{\gamma}}$ be the graph obtained from $K$ by 
removing all boxes and breaking any edge corresponding to $i \in \underline{\gamma}-\underline{\varepsilon}_{\phi}$. For example, with $K$ as above:

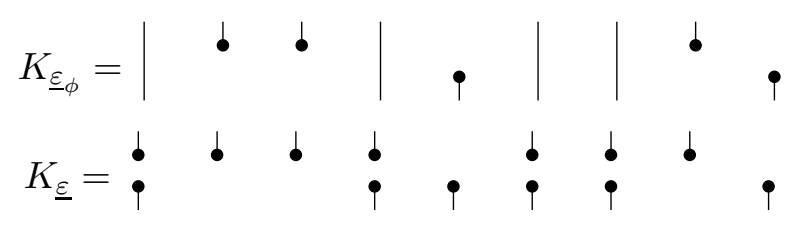

On the other hand, let $M$ be the $R$-algebra with generators $A_{s}$ and $B_{s}$ for $s \in S$ and "nil Hecke" relation

$$
A_{s} f=s(f) A_{s}+\partial_{s}(f) B_{s}
$$

for $s \in S$ and $f \in R$. Define $F=F_{1} \cdots F_{m} \in M$, where

$$
F_{i}= \begin{cases}\alpha_{s_{i}} & \text { if } e_{i}^{1}=e_{i}^{2}=U 0 \\ B_{s_{i}} & \text { if exactly one of } e_{i}^{1} \text { and } e_{i}^{2} \text { is } U 0 \\ A_{s_{i}} & \text { otherwise }\end{cases}
$$

For any $\underline{\gamma}$ with $\underline{\varepsilon}_{\phi} \subset \underline{\gamma} \subset \underline{\varepsilon}$, we define $F_{\underline{\gamma}}=F_{1, \underline{\gamma}} \cdots F_{m, \underline{\gamma}}$, where

$$
F_{i, \underline{\gamma}}= \begin{cases}B_{s_{i}} & \text { if } i \in \underline{\gamma} \\ A_{s_{i}} & \text { if } i \in \underline{\varepsilon}-\underline{\gamma} \\ 1 & \text { otherwise. }\end{cases}
$$

Using (5.4) we may write

$$
F=\sum_{\underline{\varepsilon}_{\phi} \subset \underline{\gamma} \subset \underline{\varepsilon}} a_{\underline{\gamma}} F_{\underline{\gamma}} \quad \text { with } a_{\underline{\gamma}} \in R
$$

Because the nil Hecke relation holds in $\mathbb{S D}$, we have

$$
G_{\underline{w^{\prime}}} \circ K \circ \bar{G}_{\underline{w^{\prime}}}=\sum_{\underline{\varepsilon}_{\phi} \subset \underline{\gamma} \subset \underline{\varepsilon}} a_{\underline{\gamma}} G_{{\underline{w^{\prime}}}^{1}} \circ K_{\underline{\gamma}} \circ \bar{G}_{{\underline{w^{\prime}}}^{2}} .
$$

Let $K_{\underline{\gamma}}^{\prime}$ be the subgraph of $K_{\underline{\gamma}}$ consisting of only unbroken vertical edges. If $\underline{w}_{\underline{\gamma}}^{\prime}$ denotes the subexpression of $\underline{w}$ corresponding to those $i \in \underline{\varepsilon}-\underline{\gamma}$, then 
$K_{\underline{\gamma}}^{\prime}$ is the identity on $B_{\underline{w}_{\gamma}^{\prime}}$. By Proposition 4.1.

$$
G_{\underline{w^{\prime}}} \circ K_{\underline{\gamma}} \circ \bar{G}_{\underline{w^{\prime}}}= \begin{cases}G_{\underline{w}^{\prime}} \circ K_{\underline{\gamma}}^{\prime} \circ \bar{G}_{\underline{w}_{\underline{\gamma}}^{\prime}} & \text { if }\left(\underline{w^{\prime}}\right)_{*}=x \\ 0 & \text { otherwise. }\end{cases}
$$

On the other hand we claim:

$$
G_{\underline{w}_{\underline{\gamma}}^{\prime}} \circ K_{\underline{\gamma}}^{\prime} \circ \bar{G}_{\underline{w}_{\underline{\gamma}}^{\prime}}= \begin{cases}\mathrm{id}_{\mathrm{x}} & \text { if } \underline{w}_{\underline{\gamma}}^{\prime} \text { is reduced } \\ 0 & \text { otherwise. }\end{cases}
$$

Indeed, if $\underline{w}_{\underline{\gamma}}^{\prime}$ is reduced then $G_{\underline{w^{\prime}}}, K_{\underline{\gamma}}^{\prime}$ and $\bar{G}_{\underline{w}_{\underline{\gamma}}^{\prime}}$ are all canonical isomorphisms between different representatives for $x$, and if $\underline{w}_{\gamma}^{\prime}$ is not reduced then $G_{\underline{w^{\prime}}} \circ K_{\underline{\gamma}}^{\prime} \circ \bar{G}_{\underline{w}_{\underline{\gamma}}^{\prime}}$ is an endomorphism of $x$ of negative degree, and $\operatorname{End}_{\mathbb{S} \mathcal{D} \geq \mathrm{x}}(\mathrm{x})=\mathrm{R}$ is zero in negative degree. Thus

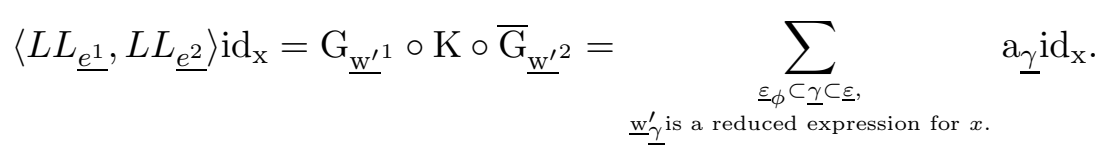

We now explain how the right hand side can be computed in the nil Hecke ring. Consider the homomorphism of $Q$-algebras

$$
p: M \rightarrow N H, \quad A_{s} \mapsto D_{s}, B_{s} \mapsto 1
$$

If $\underline{\varepsilon}_{\phi} \subset \underline{\gamma} \subset \underline{\varepsilon}$ then $\underline{w}_{\gamma}^{\prime}$ is a subexpression of both $\underline{w}^{\prime 1}$ and $\underline{w}^{\prime 2}$. In particular $\left(\underline{w}_{\gamma}^{\prime}\right)_{*} \leq \underline{w}_{*}^{\prime 1}=\underline{w}_{*}^{\prime 2}=\bar{x}$ and hence

$$
p\left(F_{\underline{\gamma}}\right) \in N H_{\leq x}:=\bigoplus_{y \leq x} R D_{y}
$$

Furthermore, in the quotient $N H_{\leq x} / N H_{<x}\left(N H_{<x}:=\bigoplus_{y<x} R D_{y}\right)$ we have

$$
p\left(F_{\underline{\gamma}}\right)= \begin{cases}D_{x} & \text { if } \underline{w}_{\underline{\gamma}}^{\prime} \text { is a reduced expression for } x \\ 0 & \text { otherwise }\end{cases}
$$


Hence applying $p$ to (5.5) we obtain

$$
p(F)=\sum_{\substack{\underline{\varepsilon}_{\phi} \subset \underline{\gamma} \subset \underline{\varepsilon}, \underline{w}_{\underline{\varepsilon}}^{\prime} \text { is a reduced expression for } x .}} a_{\underline{\gamma}} D_{x}+N H_{<x}=\left\langle L L_{\underline{e^{1}}}, L L_{\underline{e^{2}}}\right\rangle D_{x}+N H_{<x} .
$$

From the definitions we see $p(F)=f\left(\underline{e}^{1}, \underline{e}^{2}\right)$ and the result follows.

\section{Examples}

6.1. This example comes from [16, Example 8.3.1].

Let $W=S_{8}$. Let

$$
\begin{aligned}
\underline{w} & =\left(s_{1}, s_{3}, s_{2}, s_{4}, s_{3}, s_{5}, s_{4}, s_{3}, s_{2}, s_{1}, s_{6}, s_{7}, s_{6}, s_{5}, s_{4}, s_{3}\right), \\
\underline{e} & =(U 1, U 1, U 0, U 1, U 1, U 1, U 1, U 1, U 0, D 0, U 0, U 1, U 0, D 0, D 0, D 0) .
\end{aligned}
$$

Then

$$
f(\underline{e}, \underline{e})=D_{1} D_{3} \alpha_{2} D_{4} D_{3} D_{5} D_{4} D_{3} \alpha_{2} D_{1} \alpha_{6} D_{7} \alpha_{6} D_{5} D_{4} D_{3}=2 D_{x},
$$

where $x=s_{1} s_{3} s_{4} s_{3} s_{5} s_{4} s_{3} s_{7}$.

Notice that $\underline{e}$ is the unique 01 -sequence of defect 0 with $(\underline{w} \underline{e}) \bullet=x$. Also note that no $D 1$ appears in $\underline{e}$. Thus by Theorem $\left[5.1,\left\langle L L_{\underline{w}, \underline{e}}, L L_{\underline{w}, \underline{e}}\right\rangle=2\right.$. This tells that the character of $b_{w}$ in the Hecke algebra is not that predicted by Kazhdan-Lusztig theory if the characteristic of $\mathbb{k}$ is 2 .

In fact, the reducibility of the characteristic cycle shown in 16, Example 8.3.1] is implied by the above calculation, using the results of [20].

6.2. The following two examples were discovered by Braden $[21$, Appendix $\mathrm{A}]$.

6.2.1. Let $W=S_{8}$. Let

$$
\begin{aligned}
\underline{w} & =\left(s_{3}, s_{2}, s_{1}, s_{5}, s_{4}, s_{3}, s_{2}, s_{6}, s_{5}, s_{4}, s_{3}, s_{7}, s_{6}, s_{5}\right) \\
\underline{e} & =(U 1, U 1, U 0, U 1, U 0, U 1, D 0, U 1, U 1, U 0, D 0, U 0, D 0, D 0) .
\end{aligned}
$$

Then

$$
f(\underline{e}, \underline{e})=D_{3} D_{2} \alpha_{1} D_{5} \alpha_{4} D_{3} D_{2} D_{6} D_{5} \alpha_{4} D_{3} \alpha_{7} D_{6} D_{5}=2 D_{x},
$$


where $x=s_{2} s_{3} s_{2} s_{5} s_{6} s_{5}$. Again $\underline{e}$ is the unique defect zero subexpression of $\underline{w}$ such that $(\underline{w} \underline{e}) \bullet=x$.

6.2.2. We repeat the example in $D_{4}$ considered in $\S 2.10$, this time using our formula. Let $W=D_{4}$ with $S=\{s, t, u, v\}$ and such that $s u=u s, s v=$ $v s, u v=v u$. Let

$$
\underline{w}=(s, u, v, t, s, u, v),
$$

Then there are three subexpressions of $\underline{w}$ for $x=s u v$ of defect zero:

$$
\begin{aligned}
& \underline{e}^{1}=(U 0, U 1, U 1, U 0, U 1, D 0, D 0) \\
& \underline{e}^{2}=(U 1, U 0, U 1, U 0, D 0, U 1, D 0) \\
& \underline{e}^{3}=(U 1, U 1, U 0, U 0, D 0, D 0, U 1)
\end{aligned}
$$

we have

$$
\begin{gathered}
f\left(\underline{e}^{1}, \underline{e}^{1}\right)=\alpha_{s} D_{u} D_{v} \alpha_{t} D_{s} D_{u} D_{v}=0 \\
f\left(\underline{e}^{1}, \underline{e}^{2}\right)=D_{v} \alpha_{t} D_{s} D_{u} D_{v}=-D_{x} .
\end{gathered}
$$

By symmetry, the matrix $\left(\left\langle L L_{e^{i}}, L L_{e^{j}}\right\rangle\right)_{1 \leq i, j \leq 3}$ is given by

$$
\left(\begin{array}{ccc}
0 & -1 & -1 \\
-1 & 0 & -1 \\
-1 & -1 & 0
\end{array}\right)
$$

6.2.3. This example was discovered by the second author $[23]$.

Let $W=S_{12}=\left\langle s_{1}, s_{2}, \ldots, s_{9}, s_{a}, s_{b}\right\rangle$. Consider $\underline{w}$ and $\underline{e}$ as follows:

$$
\begin{aligned}
& \left(s_{1}, s_{2}, s_{1}, s_{3}, s_{2}, s_{1}, s_{5}, s_{4}, s_{6}, s_{5}, s_{4}, s_{3}, s_{7}, s_{6}, s_{5}, s_{4}, s_{3}, s_{8}, s_{7}, s_{9}, s_{8}, s_{7}, s_{6}, s_{5}\right. \text {, } \\
& \left.s_{a}, s_{b}, s_{a}, s_{9}, s_{8}, s_{7}\right) \\
& \text { (U1 U1 U1 U1 U1 U1 U1 U0 U1 U1 U1 U1 U1 U1 U1 U1 U1 U1 U1 U1 } \\
& \text { U1 U1 U0 D0 U0 U1 U0 D0 D0 D0) }
\end{aligned}
$$

Then $\underline{e}$ is the unique defect zero subexpression for

$$
x=s_{1} s_{2} s_{1} s_{3} s_{2} s_{1} s_{5} s_{6} s_{5} s_{4} s_{3} s_{7} s_{6} s_{5} s_{4} s_{3} s_{8} s_{7} s_{9} s_{8} s_{7} s_{6} .
$$


Then

$$
f(\underline{e}, \underline{e})=D_{1213215} \alpha_{4} D_{65437654387987} \alpha_{6} D_{5} \alpha_{a} D_{b} \alpha_{a} D_{987}=2 D_{x}
$$

The significance of this example is that it is probably the first example in type $A$ where $\underline{w}_{\text {. and }} x$ lie in the same two-sided cell.

\section{Acknowledgments}

We would like to thank Ben Elias, Thorge Jensen, Nicolas Libedinsky and Britta Späth for useful remarks.

\section{References}

1. M. Dyer, Representation theories from Coxeter groups, Representations of groups (Banff, AB, 1994), 105-139, CMS Conf. Proc., 16, Amer. Math. Soc., Providence, RI, 1995.

2. B. Elias, Quantum Satake in type A: part I, Preprint. arXiv: 1403.5570.

3. B. Elias, The two-color Soergel calculus, Compos. Math. 152 (2016), no. 2, 327-398.

4. B. Elias and M. Khovanov, Diagrammatics for Soergel categories. Int. J. Math. Math. Sci. 2010, Art. ID 978635, 58 pp.

5. B. Elias and G. Williamson, The Hodge theory of Soergel bimodules, Ann. Math. 180 (2014), 1089-1136. arxiv:1212.0791.

6. B. Elias and G. Williamson, Soergel calculus, to appear in Represent. Theory. arXiv:1309.0865.

7. B. Elias and G. Williamson, Diagrammatics for Coxeter groups and their braid groups, to appear in Quantum Topology, arXiv:1405.4928.

8. P. Fiebig, Sheaves on affine Schubert varieties, modular representations, and Lusztig's conjecture. J. Amer. Math. Soc. 24 (2011), no. 1, 133-181.

9. X. He, A subalgebra of 0-Hecke algebra, J. Algebra 322 (2009), 4030-4039.

10. J. E. Humphreys, Reflection groups and Coxeter groups, Cambridge Studies in Advanced Mathematics, Cambridge University Press (1990).

11. L. T. Jensen and G. Williamson, The p-canonical basis for Hecke algebras, to appear in Perspectives in categorification, arXiv:1510.01556.

12. V. G. Kac, Infinite Dimensional Lie Algebras, 3rd edn. Cambridge University Press, Cambridge (1990). 
13. B. Kostant, S. Kumar, The nil Hecke ring and cohomology of $G / P$ for a Kac-Moody group G. Adv. in Math. 62 (1986), no. 3, 187-237.

14. N. Libedinsky, Sur la catégorie des bimodules de Soergel. J. Algebra 320 (2008), no. 7, 2675-2694.

15. G. Lusztig, Hecke algebras with unequal parameters, CRM Monograph Series, 18. American Mathematical Society, Providence, RI, 2003.

16. M. Kashiwara and Y. Saito, Geometric construction of crystal bases, Duke Math. J. 89 (1997), 9-36.

17. S. Riche and G. Williamson, Tilting modules and the anti-spherical quotient, preprint, arXiv:1512.08296.

18. W. Soergel, On the relation between intersection cohomology and representation theory in positive characteristic. Commutative algebra, homological algebra and representation theory (Catania/Genoa/Rome, 1998). J. Pure Appl. Algebra 152 (2000), no. 1-3, 311-335.

19. W. Soergel, Kazhdan-Lusztig-Polynome und unzerlegbare Bimoduln über Polynomringen, J. Inst. Math. Jussieu 6 (2007), 501-525.

20. K. Vilonen, G. Williamson, Characteristic cycles and decomposition numbers, Math. Res. Lett. 20 (2013), no. 2, 359-366.

21. G. Williamson (with an appendix by Tom Braden), Modular intersection cohomology complexes on flag varieties. Math. Z. 272 (2012), no. 3-4, 697-727.

22. G. Williamson, Schubert calculus and torsion, Preprint. arXiv:1309.5055.

23. G. Williamson, A reducible characteristic variety in type A, preprint. arXiv:1405.3479. 